\title{
Détection d'espèces par spectroscopie photoacoustique : principe et applications
}

La spectroscopie photoacoustique est une technique sensible, qui permet la réalisation d'instruments compacts pour la détection de gaz à l'état de traces permettant de répondre aux besoins de diverses applications, aussi bien environnementales que de sûreté et de sécurité.

\footnotetext{
$\gg$ Myriam RAYBAUT,

Margaux BARBIER,

Jean-Michel MELKONIAN,

Jean-Baptiste DHERBECOURT,

Antoine GODARD,

Michel LEFEBVRE

Onera, The French aerospace lab,

Département Mesures Physiques

myriam.raybaut@onera.fr

\section{L'effet photoacoustique : \\ historique}

En 1880, Alexander Graham Bell découvrait I'effet photoacoustique dans les solides [1], puis les gaz. L'observation est simple : le rayonnement solaire, bloqué périodiquement, focalisé dans des objets, permet l'émission d'ondes sonores audibles à la fréquence de la modulation. De cette observation ont découlé plusieurs applications: I'invention du photophone (figure 1), ancêtre des télécommunications optiques, sans fil, dès 1880 , ou par exemple la détermination des propriétés absorbantes de l'objet émetteur, en fonction de la longueur d'onde reçue, ou spectroscopie photoacoustique [2].

Le principe général est le suivant (figure 2) : une onde lumineuse, de fréquence $v$, traverse un gaz, faisant passer les molécules de ce gaz dans un état excité, sous réserve que hv corresponde à une transition pour cette espèce moléculaire. Suite à cette transition, les molécules excitées peuvent retourner à leur
}

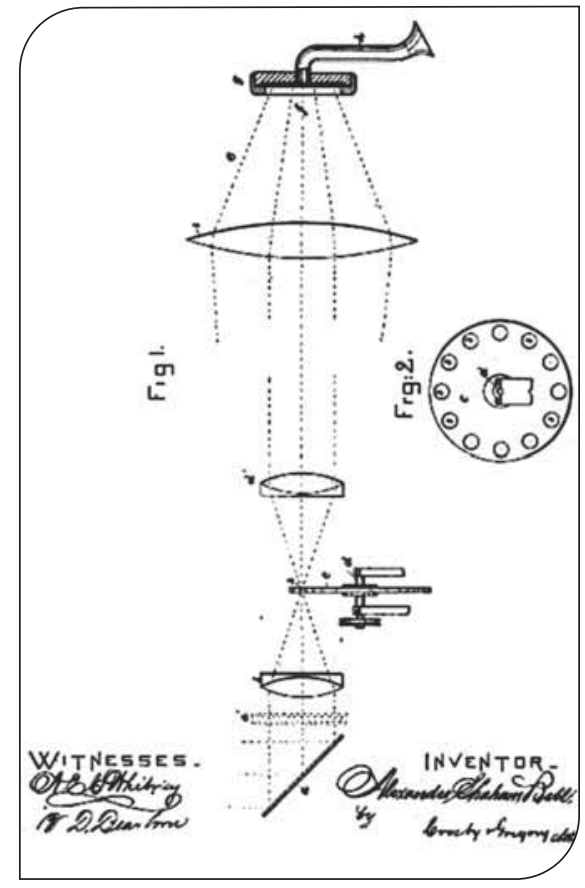

Figure 1. Schéma de principe du photophone utilisant l'effet photoacoustique, inventé par G. Bell en 1880 .

état fondamental en dissipant l'énergie absorbée par collision vers les molécules voisines, donc sous forme de chaleur. On observe ainsi un échauffement local transitoire, qui donne naissance à une onde de pression lorsque l'onde lumineuse est modulée. Cette onde de pression peut être détectée par un microphone. Il est ainsi possible, par analyse de la réponse acoustique en fonction de la longueur d'onde d'excitation, d'obtenir le spectre d'absorption de la molécule. Cette technique a fait l'objet de progrès significatifs au cours des dernières $\gg$
Optique adaptative en mitroseopite mi

Le miroir déformable mirao 52-e, avec ou sans analyseur de surface d'onde HASO, avec nos logiciels CASAO OU GENAO, offire la meilleure capacité de correction ot permet d'améliorer la résolution de l'image de manière spectaculaire.

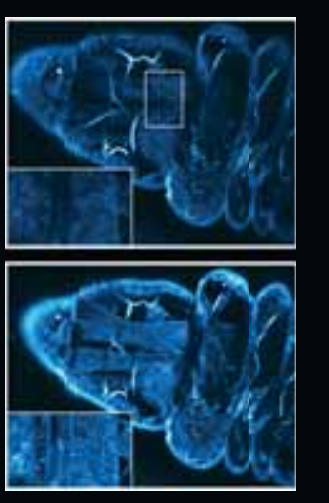

En haut, image d'une larve de drosophile en microscopie THG non-corrigée. En bas, image du même échantillon corrigée avec l'optique adaptative. Images de E. Beaurepaire, D. Débarre et N. Olivier, Ecole Polytechnique, LOB

Venez nous rencentrer sur notre stand L37 à PRI-OPTO du 4 au 6 octobre à Paris, Porte de Versailles.

Pour plus d'informations, appelez +33 (0)1 6486 15 60, ou visitez: imagine-optic.oom

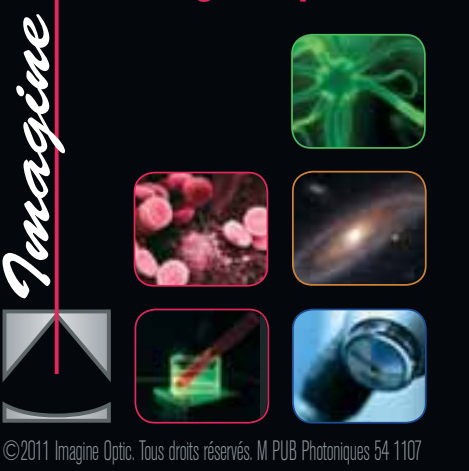




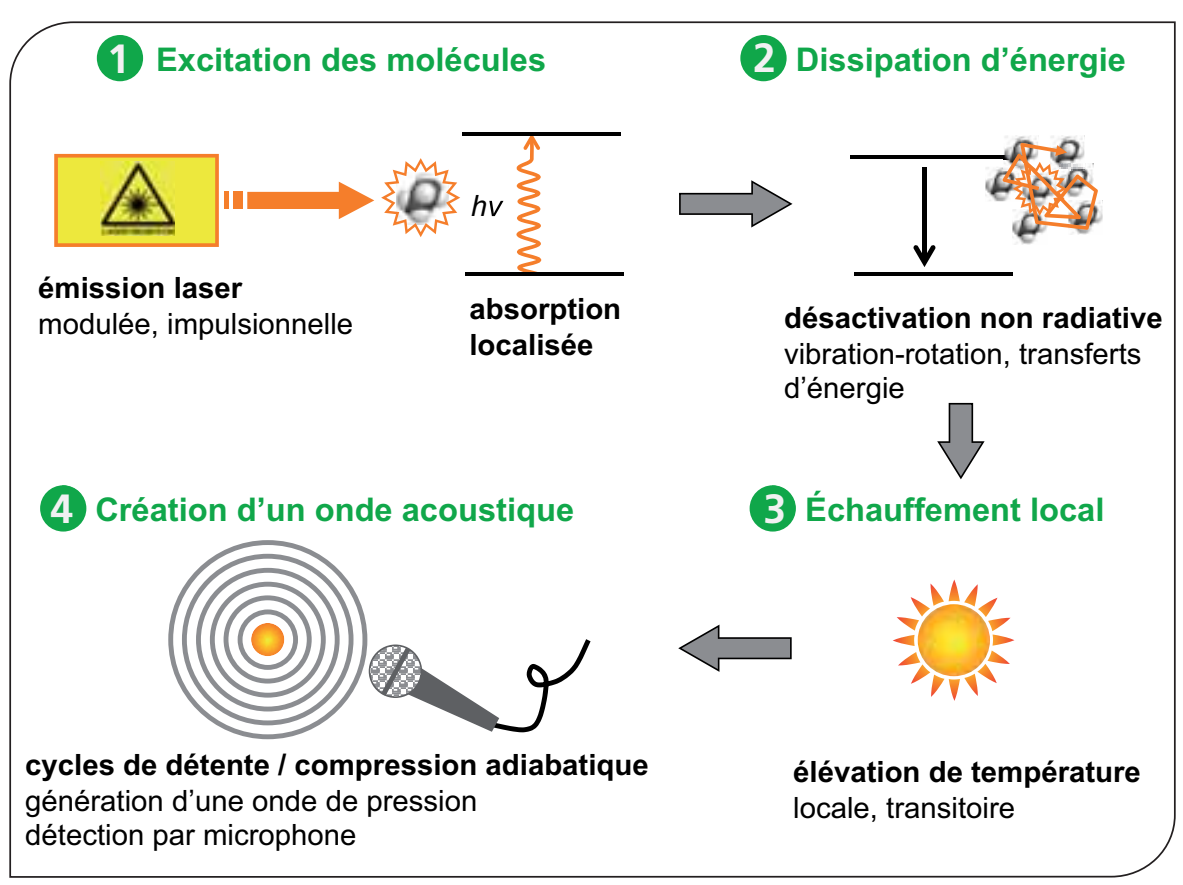

Figure 2. Principe de l'effet photoacoustique : le faisceau lumineux modulé (ou impulsionnel) est focalisé, une onde sonore est générée et détectée.

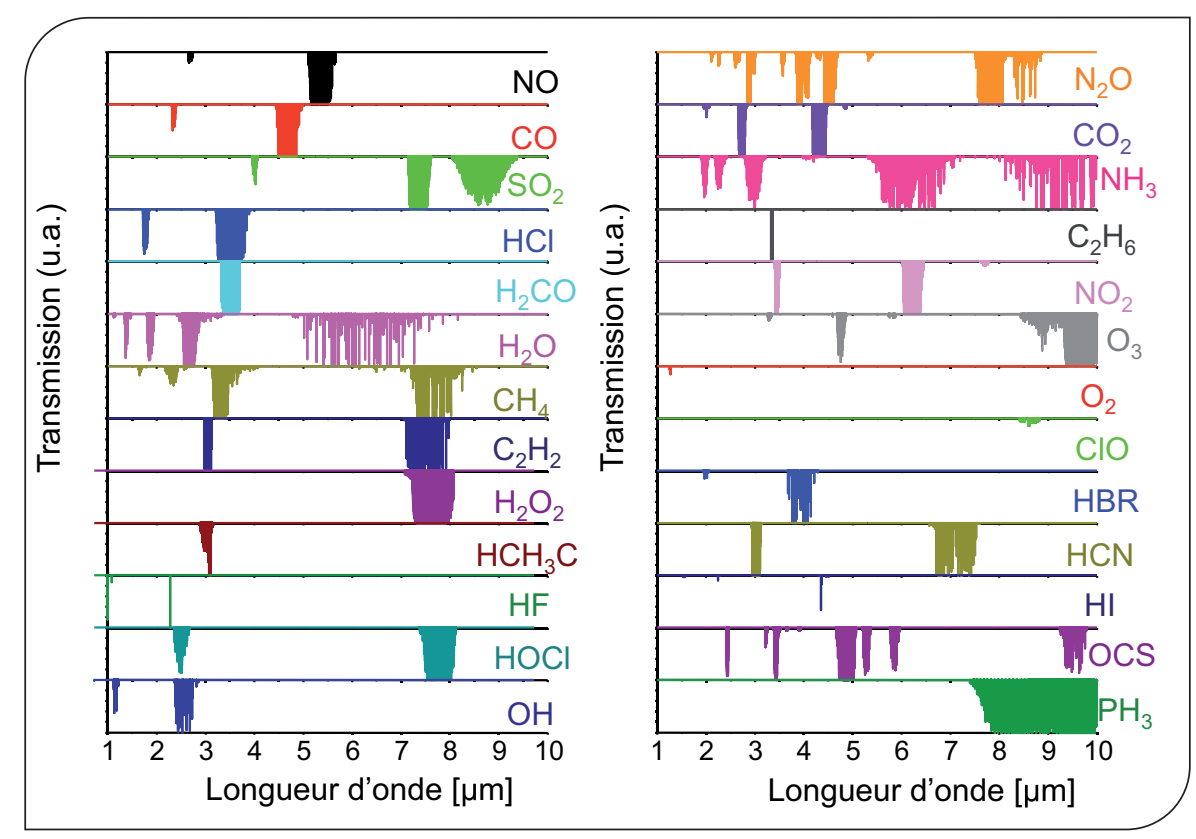

Figure 3. Spectre d'absorption infrarouge de diverses espèces polluantes et composés organiques volatils.

décennies. Elle a progressivement bénéficié des avancées majeures en termes de sources optiques (invention du laser [3], puis des sources non linéaires, des diodes lasers, des lasers à cascade quantique...), de microphones et d'électronique, et permet aujourd'hui la détection de gaz à l'état de traces et le développement d'instruments adaptés à diverses applications. comme nous le verrons ci-après; de plus, elle est «large bande» : une cellule acoustique peut être conçue quelle que soit la longueur d'onde d'excitation, contrairement aux méthodes de spectroscopie par absorption, qui nécessitent des traitements diélectriques spécifiques (cavités multi-passages) et/ou des détecteurs optiques adaptés à la gamme spectrale d'émission de la source utilisée.

La technique photoacoustique repose sur la génération et la détection efficace de I'onde acoustique. La réalisation d'un instrument photoacoustique performant nécessite donc une optimisation conjointe des performances de la source lumineuse et du système de détection acoustique, adaptés à l'espèce à détecter.

\section{Optimisation de la source Iumineuse}

L'intensité du signal acoustique généré est tout d'abord fonction de la concentration de l'espèce et de son absorbance à la longueur d'onde d'excitation. Elle est aussi fonction de la puissance lumineuse incidente.

La source doit être capable d'adresser la gamme spectrale des raies d'absorption de la molécule étudiée avec des propriétés spectrales compatibles avec une absorption significative de l'onde lumineuse. La gamme spectrale infrarouge $(1,5$ à $12 \mu \mathrm{m})$ est, en particulier, très pertinente car la plupart des molécules intéressantes présentent des raies d'absorption les plus intenses et bien distinctes dans cette région dite molecular fingerprint region (figure 3). De plus, il est nécessaire de moduler temporellement l'absorption des molécules afin de produire l'onde sonore via une succession de séquences d'excitation/relaxation. Cela peut être réalisé par modulation d'intensité de la source (source continue modulée ou source fonctionnant en régime impulsionnel), ou par modulation en longueur d'onde (figure 4). Dans ce dernier cas, I'amplitude de modulation doit être adaptée à la largeur de la raie d'absorption balayée. L'agilité en fréquence, ainsi que la finesse spectrale de la source, sont particulièrement critiques 


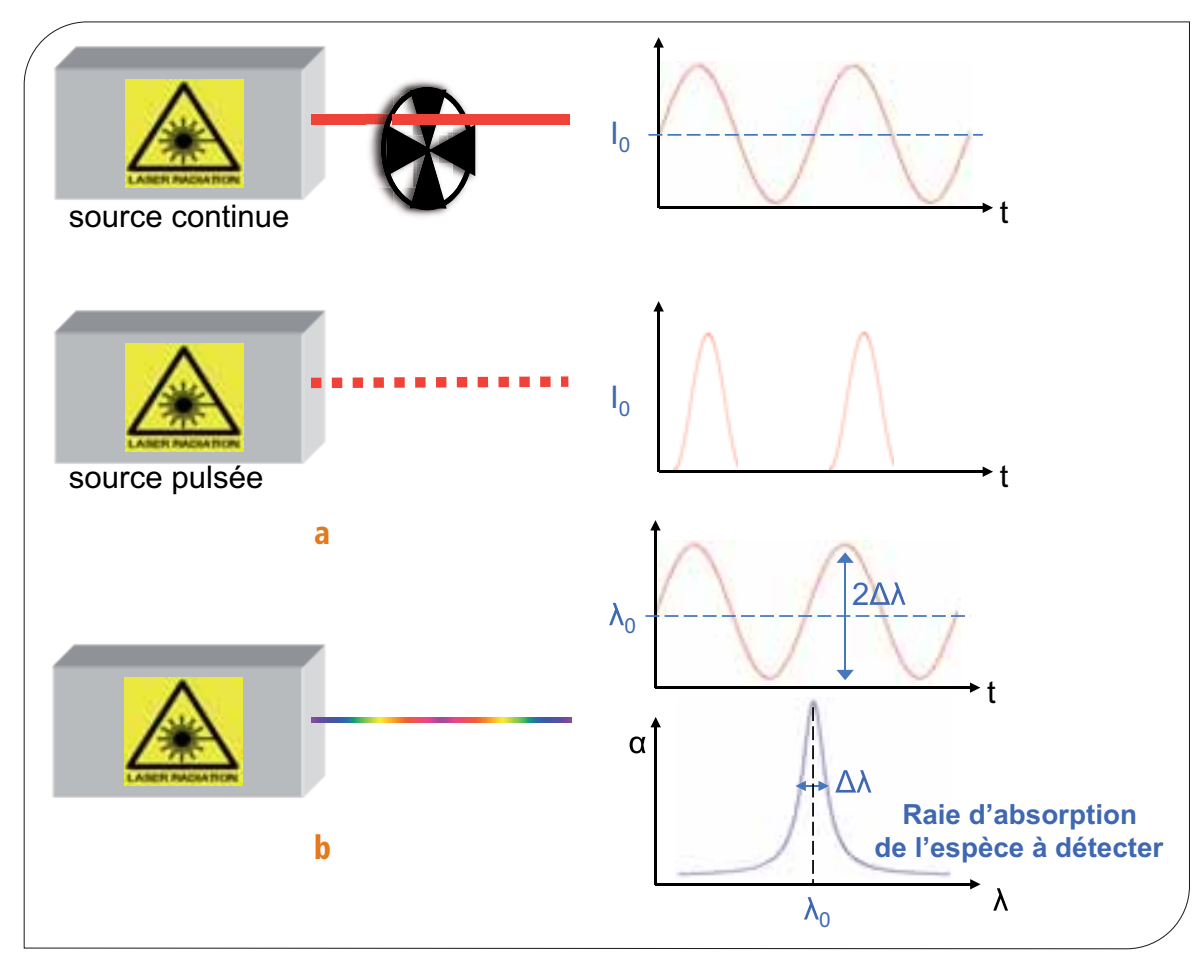

Figure 4. Schémas des possibilités de modulation de la source : en amplitude avec une source continue modulée ou une source impulsionnelle (a), en longueur d'onde (b).

dans le cas de mélanges, afin de discriminer l'espèce recherchée des interférents.

\section{Optimisation de l'architecture de détection acoustique}

Pour une meilleure détection de l'onde sonore, deux architectures principales ont été étudiées et réalisées (figure 5) : - l'utilisation d'une cavité résonante pour l'onde sonore, de facteur de qualité $Q_{c a v}$ plus ou moins élevé, de fréquence de résonance adaptée à la cadence de modulation ou à la cadence de répétition de la source, couplée à un microphone [4] ;

- I'utilisation de diapasons en quartz comme détecteurs acoustiques dont la sensibilité peut être qualifiée par un facteur de qualité du diapason noté $Q_{\text {dia }}$ [5].

\section{Cellules acoustiques résonantes}

L'utilisation de cellules acoustiques résonantes est aujourd'hui la plus éprouvée des deux techniques.

Àla résonance de la cellule, on peut écrire l'amplitude de l'onde sonore générée en fonction d'une "constante de cellule", qui décrit la sensibilité du résonateur à une fréquence donnée. Cette constante de cellule est fonction de différents paramètres : fréquence, facteur de qualité, dimensions de la cavité, recouvrement spatial entre le faisceau laser et le mode acoustique.

La qualité du ou des microphones, leur positionnement sur un ventre de champ ainsi que les performances de l'électronique d'amplification et d'acquisition sont aussi des paramètres importants pour la réalisation d'instruments de bon-

Des architectures de cellules plus ou moins complexes et compactes peuvent être envisagées, afin de détecter le signal de manière optimale et de répondre notamment aux problématiques de bruit propres à cette technique. Par exemple, tout phénomène $d$ 'absorption par un matériau autre que le gaz ou mélange à analyser, comme les fenêtres ou parois de la cellule, peut se traduire par un signal de "fond", synchrone avec la fréquence d'excitation, qui peut être amplifié comme le signal utile à la résonance de la cellule. L'utilisation de cavités tampon ou buffer peut limiter le niveau de ce fond. Aussi, I'utilisation d'une méthode différentielle, basée sur l'utilisation de deux microphones, permet $\gg$ ne détectivité.

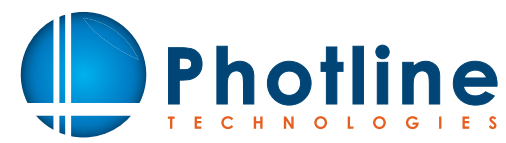

Delivering Modulation Solutions
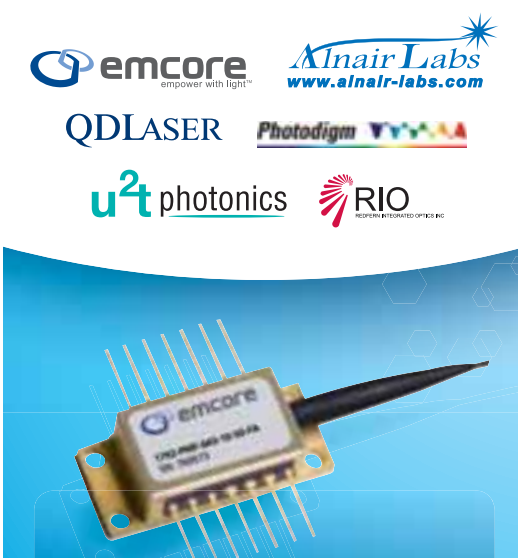

:t Lasers

- Accordables bande C, bande L

- DFB faible RIN jusqu'à $100 \mathrm{~mW}$

- Spectre étroit : jusqu'à $3 \mathrm{kHz}$

- 780 nm, 850 nm, 1053 nm,

- $1064 \mathrm{~nm}, 1300 \mathrm{~nm}, 1550 \mathrm{~nm}$

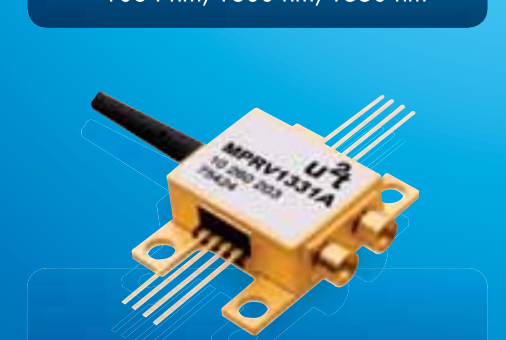

:: Photodétecteurs

- $10 \mathrm{GHz}, 20 \mathrm{GHz}, 40 \mathrm{GHz}$, $70 \mathrm{GHz}, 100 \mathrm{GHz}$

- Numériques, analogiques

- Equilibrés pour détection cohérente

- Récepteurs intégrés DPSK, DQPSK

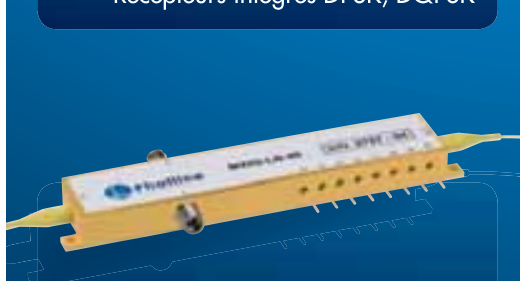

:i: Modulateurs $\mathrm{LiNbO}_{3}$

- Intensité et phase

- Jusqu'à $40 \mathrm{~Gb} / \mathrm{s}$ - $40 \mathrm{GHz}$

- $1550 \mathrm{~nm}, 1310 \mathrm{~nm}$

- 800 nm, 1000 nm, 2 rm

contact@photline.com

Tél. : 0381853180

www.photline.com 


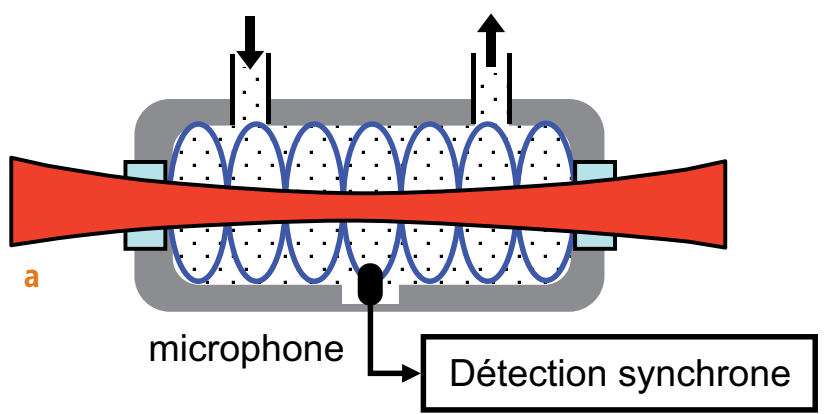

Figure 5. Exemples d'architectures de résonateurs acoustiques : cellule photoacoustique linéaire (a) , cellule photoacoustique différentielle avec buffers acoustiques et filtres quart d'onde (b) ; exemples de diapasons en quartz sans ou avec micro-résonateur (c) et (d).

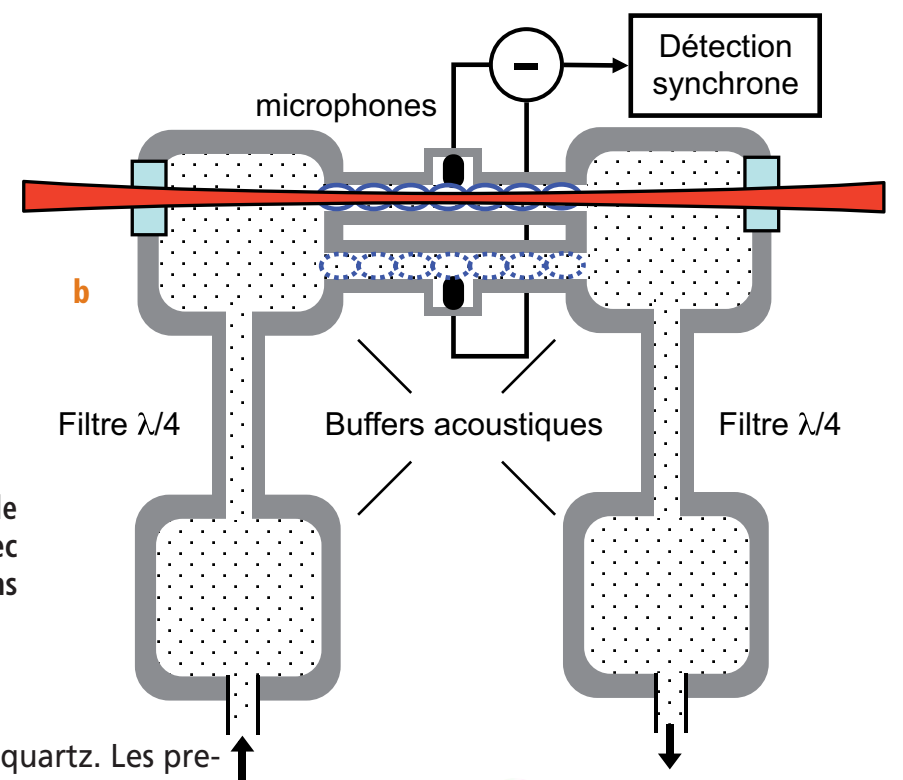

de diminuer le bruit électrique lié au bruit acoustique ambiant, aux possibles perturbations électromagnétiques externes, ou bien éventuellement au flux du gaz dans la cellule [4].

Des instruments performants pour la détection de diverses espèces ont ainsi été présentés dans la littérature : ils sont basés sur le couplage de diverses sources infrarouges et cellules acoustiques résonantes. La détection de gaz à l'état de traces est ainsi possible, et nous pouvons par exemple noter que des détectivités de 10 ppt (parties dans $10^{12}$ ) sur éthylène ont été obtenues avec une excitation par laser $\mathrm{CO}_{2}, 10$ ppt sur l'éthane avec une excitation par OPO [6], quelques dizaines de ppb (parties dans $10^{9}$ ) sur NO avec une source laser à cascade quantique [7], quelques ppb sur l'ammoniac avec une diode laser [8], et quelques ppb pour le formaldéhyde avec une source OPO (Oscillateur Paramétrique Optique) $[6,8]$.

\section{Diapasons en quartz}

L'utilisation des diapasons en quartz comme méthode de détection de l'onde acoustique est une alternative récente, qui a donné lieu à plusieurs travaux depuis une dizaine d'années. Cette technique ouvre la voie au développement de capteurs plus compacts. Elle permet une bonne sélectivité spatiale et une bonne immunité au bruit ambiant puisque la plupart des quartz utilisés sont des diapasons de fréquence de vibration propre autour de $32,8 \mathrm{kHz}$ développés pour les horloges à quartz. Les pre- $\mathbf{\uparrow}$ mières études ont permis de démontrer rapidement des concentrations équivalentes au bruit inférieures au ppm (parties dans $10^{6}$ ) sur $\mathrm{NH}_{3}, \mathrm{~N}_{2} \mathrm{O}$, $\mathrm{CO}, \mathrm{CH}_{2} \mathrm{O}$ [5]. Récemment, de nouvelles architectures couplant le diapason avec un micro-résonateur (appelées « spectrophones») ont permis de détecter par exemple, quelques ppm de $\mathrm{H}_{2} \mathrm{~S}$ [9], quelques centaines de ppb d'éthylène [10] et quelques dizaines de ppb de fréon [11]. Ces nouvelles architectures ouvrent, sans nul doute, la voie vers le développement $d$ 'instruments performants et fortement intégrés, répondant aux besoins de diverses applications.

\section{Applications}

Le besoin d'instruments permettant l'identification, la quantification ou le monitoring d'espèces chimiques à l'état de traces est un besoin générique couvrant un large domaine d'applications. Nous pouvons relever, parmi les réalisations récentes, des applications en biologie, telles que le suivi du processus de germination ou de la maturation des fruits pour les zones de stockage de ces denrées. Dans ce cas, des instruments permettant le monitoring de l'éthylène ou du méthane [8] sont nécessaires.

En médecine, le développement d'instruments permettant des diagnostics non invasifs est sollicité, par exemple, pour l'analyse de l'air expiré. Dans ce cas, les biomarqueurs de certaines pathologies

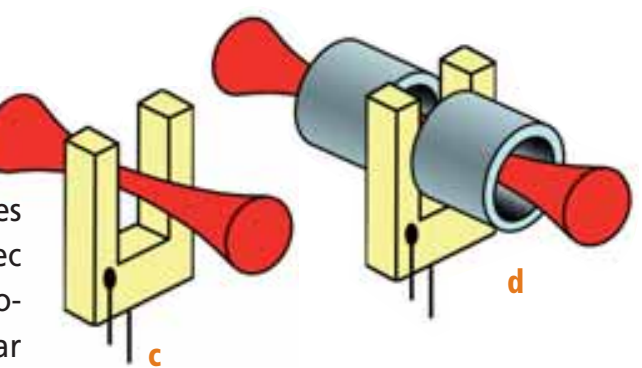

peuvent être suivis, tels que l'éthylène pour I'hypertension ou les dommages dermatologiques sous exposition UV [6] ou NO pour mesurer le degré d'inflammation de l'asthme [6], [7]. La détection d'agents dopants (stimulants, anabolisants, bêtabloquants...) [8] par méthode photoacoustique est aussi étudiée, ainsi que par exemple la possibilité d'un monitoring du glucose pour les patients diabétiques.

Pour des applications environnementales, le suivi des concentrations atmosphériques en gaz à effet de serre $\left(\mathrm{CO}_{2}\right.$, $\left.\mathrm{CH}_{4}, \ldots\right)$ peut être réalisé par méthode photoacoustique [12]. De même, ces méthodes peuvent permettre une mesure des émissions polluantes [6], [12] $\left(\mathrm{NO}_{x_{1}}\right.$ $\left.\mathrm{CO}_{x}, \ldots\right)$ ou des suies en sortie moteur, dans les secteurs automobiles et aéronautiques.

Enfin, le sujet humain peut être très sensible à la présence de quelques ppm voire ppb de certains composés organiques volatiles, et l'exposition à ces espèces peut être source de désagréments pour sa santé, ou même être létale. Pour des applications dans le domaine de la sûreté 
industrielle, du contrôle environnemental ou de la gestion de la santé publique, des instruments sont étudiés afin d'être à même de mesurer les émissions des polluants industriels tels que $\mathrm{NO}_{x}, \mathrm{CO}_{x_{1}}$ $\mathrm{SO}_{2}$ [12], I'ammoniac [8], ou bien des espèces chimiques ou composés volatiles organiques, telles que l'acétone, le fréon [11], $\mathrm{H}_{2} \mathrm{~S}$ [9] ou le formaldéhyde [8]. Ces développements sont nécessaires afin de disposer de capteurs performants permettant de s'assurer du respect des normes de qualité de l'air et des rejets atmosphériques, voire d'alerter en cas $d^{\prime}$ accident et de rejet invasif. D'un point de vue sécurité, I'approche photoacoustique est aussi intéressante pour la détection d'explosifs (TATP, TNT, ...) ou de leurs précurseurs (acétone...), ou bien encore d'agents toxiques (DIMP, DMMP...) [13]. Pour ces divers cas applicatifs, la photoacoustique est une technique intéressante car une approche multi-espèces peut être envisagée, et les seuils de détectivité qui peuvent être obtenus sont compatibles avec les besoins.

\section{Vers des systèmes déployés sur le terrain}

La problématique d'identification et de quantification d'une espèce minoritaire dans un mélange complexe (par exemple l'atmosphère), est donc une problématique actuelle et difficile, répondant aux besoins d'applications civiles comme le contrôle environnemental, la gestion de la santé publique voire les diagnostics médicaux, ou la réponse aux problématiques de sûreté et de sécurité. Pour répondre au mieux aux besoins de ces applications, le développement d'instruments multi-espèces doit être poursuivi, afin d'obtenir des mesures précises, avec des taux de fausses alarmes faibles. La technique photoacoustique fait partie des techniques de choix car elle permet le déploiement de capteurs locaux, avec des seuils de détectivité compatibles avec les besoins. Les développements sur cette thématique se poursuivent activement, notamment au niveau de la miniaturisation des systèmes, basés sur l'utilisation de sources compactes et de détecteurs acoustiques miniatures, et au niveau de l'analyse de mélanges gazeux, pour la détection de gaz à l'état de traces multiespèces, en présence d'interférents.

\section{Références}

[1] A.G. Bell, Am. J. Sci. 20, 35 (1880).

[2] M.L. Viengerov, Dokl. Akad. Nauk SSSR 19, 687 (1938).

[3] E.L. Kerr and J.G. Atwood, Appl. Opt. 7, 915 (1968).

[4] A. Miklos, P. Hess and Z. Bozoki, Rev. of Sci. Inst. 72, 1937 (2001).

[5] A.A. Kosterev, et al., Rev. Of Sci. Inst. 76 (2005)

[6] S.M. Cristecu et al., Appl. Phys. B 92, 343 (2008)

[7] A. Grossel et al., Infrared Physics and Technology 51, 95 (2007).

[8] Special Issue: Tunable Diode Laser Spectroscopy, Appl. Phys. B. 85, (2006)

[9] A.A. Kosterev, et al., Appl. Phys. B 101, 849 (2010)

[10] S. Shilt, et al. Appl. Phys. B 95, 813 (2009)

[11] A.A. Kosterev, et al., Appl. Phys. B 100, 173 (2010)

[12] A. Berrou, et al., Appl. Phys. B 98, 217 (2010)

[13] C.K.N. Patel, The European Physical Journal Special Topics 153, 1-18 (2008).

\section{HORIBA Scientific}

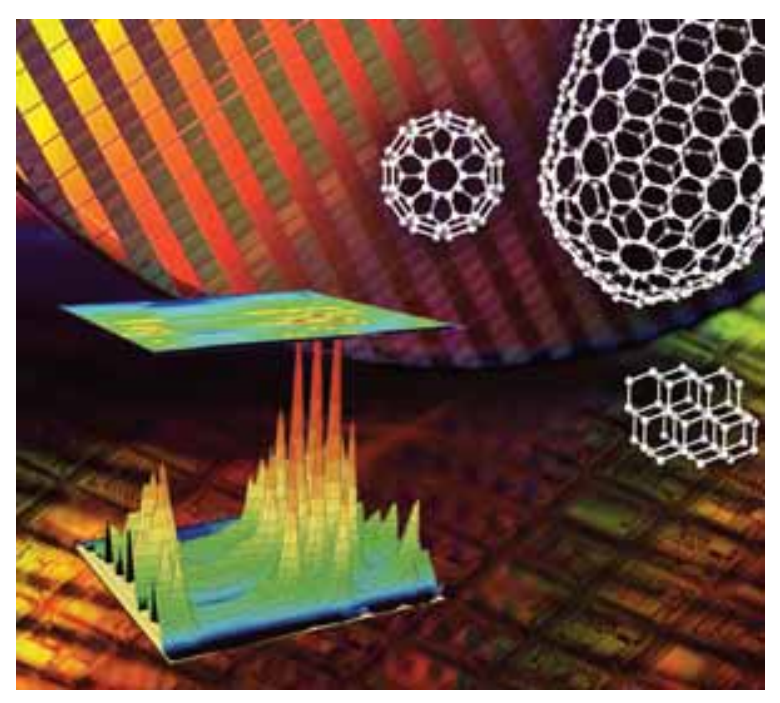

www.horiba.com/scientific

\section{Nano-réalité !}

Relevez le défi de la caractérisation des nano-structures, de la détermination des informations chimiques structurales et dimensionnelles à l'échelle du nanomètre.

\section{Spectroscopie Raman - Spectrofluorimétrie Ellipsométrie Spectroscopique Analyse de taille de particules}

Contactez-nous au +33(0)1 64541300 . 